\title{
Ferroelectricity and multiferroicity: Broader way to go beyond
}

\author{
Jun-Ming Liu ${ }^{1, *}$, Ce-Wen $\mathrm{Nan}^{2, \dagger}$ \\ ${ }^{1}$ Department of Physics, Nanjing University, Nanjing 210093, China \\ ${ }^{2}$ Department of Materials Science and Engineering, Tsinghua University, Beijing 100084, China \\ E-mail:*liujm@nju.edu.cn, ${ }^{\dagger}$ cwnan@mail.tsinghua.edu.cn \\ Received June 29, 2012; accepted July 13, 2012
}

Ferroelectrics and magnetics represent two major classes of functional materials which people find everywhere in daily life, though they are usually beyond the views of people and hidden inside the hearts of most devices. While spintronics [1] comes into being, reviving magnetism which has been as old as ferroelectricity, since decades ago the physics of ferroelectrics has turned its attention to the domain scale from the atomic level at which the physics was believed to be well understood [2]. As is well known, it is a small world, and ferroelectricity finally has its marriage with magnetism at a proper time [3], giving birth to multiferroicity, a concurrent and aggressive field of condensed matter physics where both ferroelectricity and magnetism can find their fans [4]. Consequently, multiferroicity bridges ferroelectricity and magnetism in an extraordinary manner, generating a number of novel phenomena and raising a pocket of key issues.

It should be mentioned that those rudiment concepts backing multiferroicity were introduced as early as 1950s for ferroelectromagnetism and 1970s for magnetoelectric (ME) composites [5]. Not much progress has been made until the end of the 20th century when the giant ME coupling effect in artificial ME composites was reported [6]. More than this, the first decade of the 21st century have evidenced the discovery of a number of multiferroic compounds in which the ferroelectricity is intrinsically linked with specific spin orders via coupling mechanisms probably more than the spin-orbit and spin-lattice interactions [7]. These milestones have then established multiferroicity as an emergent concept in condensed matters and promising candidates for dreams in materials science and electronic engineering.

Nevertheless, one major driving force for the rapid progress of multiferroic researches in last years is those promising applications in data storage and sensing technologies. So far, prototype devices based on various ME composite structures have been demonstrated [5], but those multiferroic compounds are still in their earlier stage in marching into any

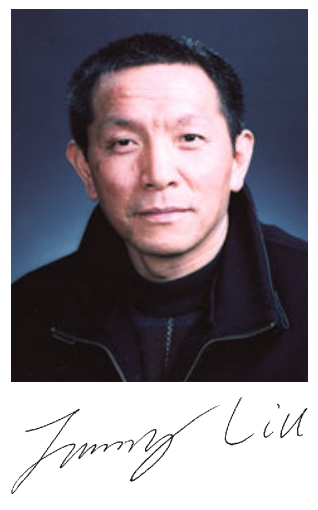

Professor Jun-Ming Liu

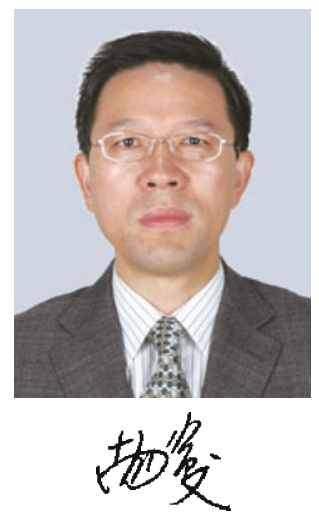

Professor Ce-Wen Nan practical applications due to a series of challenges such as low operating temperature and insufficient performances [8]. In fact, no clear scenario about the microscopic mechanism is yet available, appealing substantial effort in kicking out these issues. Currently, three parallel roadmaps are under tracking: i) technological approaches of bulk ME composites for sensing and energy harvesting, ii) ME heterostructures for electric control of magnetization for data storage and processing, and iii) understanding of fundamental physics underlying multiferroic compounds. Clearly, roadmaps ii) and iii) are far behind i).

Frontiers of Physics intends to make a contribution to this exciting research field by inviting active experts to contribute and delivers this special topic. This special topic collects eight reports uncovering one corner of multiferroic iceberg. Chun-Gang Duan presents a perspective on the state-of-arts of electric control of magnetism which is a key issue of next-generation data storage [9]. Yue-Wei Yin et al. study the ferroelectric tunneling using a scan- 
ning probe technique and multiferroic tunnel junctions using ferromagnetic $\mathrm{La}_{0.7} \mathrm{Ca}_{0.3} \mathrm{MnO}_{3}$ and $\mathrm{La}_{0.7} \mathrm{Sr}_{0.3} \mathrm{MnO}_{3}$ as the electrodes and ferroelectric $(\mathrm{Ba}, \mathrm{Sr}) \mathrm{TiO}_{3}$ as the barrier in trilayer planner junctions [10]. Masashi Tokunaga deals with the ME response of several compounds under high magnetic field, collecting highly valuable data [11]. $\mathrm{Na}$ Zhang et al. reveal the two origins for ferroelectricity generation in two magnetic species compound $\mathrm{DyMnO}_{3}$ [12]. Zhen Chen et al. investigate in details the electronic structure of multiferroic $\mathrm{YMn}_{2} \mathrm{O}_{5}$ using high-resolution TEM assisted with first-principles calculations [13]. Cheng-Liang Lu et al. address the ferroic domain coupling with substrate strain in high-quality $\mathrm{BiFeO}_{3}$ thin films [14]. Gopalan Srinivasan and his collaborator investigate the effect of interfacial details in ME composites using broadband resonance technique [15], while Shu-Hong Xie et al. develop a novel route to synthesize multiferroic nanofibres, extending the capability of multiferroic materials by incorporating size effect [16].

We do not expect this specific topic can overwhelm even one of those key issues, but it would be wonderful if it is a dot of multiferroic episode. It also sheds lights on the materials issues and physics cores which we have to handle not only for research fans and more substantially for practical applications in the near future, hopefully.

\section{References}

1. I. Žutić, J. Fabian, and S. Das Sarma, Rev. Mod. Phys., 2004, $76(2): 323$

2. A. S. Sidorkin, Domain Structure in Ferroelectrics and Related Materials, Cambridge: Cambridge University Press, 2006

3. K. M. Rabe, J. M. Triscone, and C. H. Ahn, Physics of Ferroelectrics: A Modern Perspective, Berlin: Springer-Verlag, 2007

4. K. F. Wang, J. M. Liu, and Z. F. Ren, Adv. Phys., 2009, 58(4): 321

5. C. W. Nan, M. I. Bichurin, S. X. Dong, D. Viehland, and G. Srinivasan, J. Appl. Phys., 2008, 103(3): 031101

6. M. Fiebig, J. Phys. D, 2005, 38(8): R123

7. S.-W. Cheong and M. Mostovoy, Nat. Mater., 2007, 6: 13
8. R. Ramesh and N. A. Spaldin, Nat. Mater., 2007, 6: 21

9. C. G. Duan, Front. Phys., 2012, 7(4): 375

10. Y. W. Yin, M. Raju, W. J. Hu, X. J. Weng, K. Zou, J. Zhu, X. G. Li, Z. D. Zhang, and Qi Li, Front. Phys., 2012, 7(4): 380

11. M. Tokunaga, Front. Phys., 2012, 7(4): 386

12. N. Zhang, S. Dong, and J. M. Liu, Front. Phys., 2012, 7(4): 408

13. Z. Chen, R. J. Xiao, C. Ma, Y. B. Qin, H. L. Shi, Z. W.Wang, Y. J. Song, Z. Wang, H. F. Tian, H. X. Yang, and J. Q. Li, Front. Phys., 2012, 7(4): 429

14. C. L. Lu, J. M. Liu, and T. Wu, Front. Phys., 2012, 7(4): 424

15. D. V. B. Murthy and G. Srinivasan, Front. Phys., 2012, 7(4): 418

16. S. H. Xie, Y. Y. Liu, and J. Y. Li, Front. Phys., 2012, 7(4): 399 